\title{
RE: risk factors for systemic inflammatory response syndrome following percutaneous nephrolithotomy
}

\author{
Ibrahim Halil Bozkurt • Burak Arslan · Tarik Yonguc
}

Received: 8 January 2014 / Accepted: 4 February 2014 / Published online: 15 February 2014

(C) Springer-Verlag Berlin Heidelberg 2014

\section{Dear Editor,}

We have read the paper, "Risk factors for systemic inflammatory response syndrome following percutaneous nephrolithotomy" with great interest and appreciate the work of the authors [1]. They have tried to determine the risk factors affecting the development of postoperative systemic inflammatory response syndrome (SIRS) after percutaneous nephrolithotomy (PCNL). Authors stated that they have used the terms, "sepsis" and "SIRS" according to the criteria defined by the International Sepsis Definitions Conference [2]. They have detected 53 (16.7\%) SIRS cases out of 317. Also they have reported that preoperative urine cultures (UCs), intraoperative renal pelvic urine cultures (RPUCs) and stone cultures (SCs) were positive in 33.9, 22.5 and $28.6 \%$ of patients with SIRS, respectively, which was found to be only $9.8,3.3$ and $4.2 \%$ for the corresponding specimens in the non-SIRS group. They concluded that positive preoperative UCs, intraoperative RPUCs and SCs are important factors indicating the development of postoperative SIRS. We have a few queries.

International Sepsis Definitions Conference defines "sepsis" as a clinical syndrome defined by the presence of both infection and a systemic inflammatory response. Infection is defined as a pathological process caused by invasion of normally sterile tissue or fluid or a body cavity by pathogenic microorganisms [2]. In this regard, we believe that it would be more appropriate to define the SIRS cases with positive intraoperative RPUCs and/or SCs as "patients with

I. H. Bozkurt $(\bowtie) \cdot$ B. Arslan · T. Yonguc

Izmir Bozyaka Training and Research Hospital Department of Urology, Saim Cikrikci Str. No: 59, Bozyaka, Izmir, Turkey e-mail: ihalilbozkurt@yahoo.com sepsis". Are there any another factors why the authors have used the term SIRS for these particular group of patients?

The authors have stated that 22 patients had antibiotic treatment according to urine culture results in SIRS patients. We are hopefully looking forward to be informed about which selection criteria were used for antibiotic treatment in SIRS patients as defined by the International Sepsis Definitions Conference.

We have read from the paper of Lojanapiwat et al. [3] stating that the bacteria isolated from patients with sepsis revealed more resistance to ceftriaxone, ceftazidime, quinolones and amoklavin-klavulonik asit, while being relatively more sensitive to amikacin, ampicillin, vancomycin and carbapenem. And these results guided the authors to rearrange the antibiotics used in the prophylaxis. In case of positive cultures, what was the resistance pattern of the microorganisms and does it have any impact on anticipating severe sepsis or septic shock in the current study?

Thank you for your concern,

Best regards.

Ibrahim Halil Bozkurt

Conflict of interest None.

\section{References}

1. Erdil T, Bostanci Y, Ozden E et al (2013) Risk factors for systemic inflammatory response syndrome following percutaneous nephrolithotomy. Urolithiasis 41(5):395-401

2. Levy MM, Fink MP, Marshall JC et al (2003) 2001 SCCM/ESICM/ACCP/ATS/SIS International Sepsis Definitions Conference. Crit Care Med 31:1250-1256

3. Lojanapiwat B, Kitirattrakarn P (2011) Role of preoperative and intraoperative factors in mediating infection complication following percutaneous nephrolithotomy. Urol Int 86:448-452 